\title{
Karyotype Analysis of Chinese Cherry "Bijie 7" and "Zunyi 3"
}

\author{
Li Xueou ${ }^{1,2, a}$, Wang Yan ${ }^{1,2, b}$, Wang Lei ${ }^{1,2, c}$ and Wang Xiaorong ${ }^{1,2, d}$ \\ ${ }^{1}$ College of Horticulture, Sichuan Agricultural University, Chengdu 611130, China
}

${ }^{2}$ Institute of Pomology and Olericulture, Sichuan Agricultural University, Chengdu 611130, China ª675042426@qq.com, b261030904@qq.com, 364589752@qq.com, dwangxr@sicau.edu.cn

Keywords: Chinese cherry floral buds Karyotype analysis

Abstract. In the study, floral buds of two varieties of chinese cherry "Bijie 7", "Zunyi 3" were used for chromosome staining as well as karyotype analysis. As the result shows, the chromosome number of cultivated species "Bijie 7" is 32 . The karyotype formula is $2 n=4 x=32=32 \mathrm{~m}$, the ratio of chromosome length $(\mathrm{L} / \mathrm{S})$ is 1.75 , and the constitution of the relative length is $4 \mathrm{~L}+8 \mathrm{M} 2+20 \mathrm{M} 1$. The relative lengths range from $10.17 \%-17.76 \%$, the centromeric index range from $39.58 \%-47.32 \%$, and arm ratio rank from 1.11 to 1.53 . Karyotype characteristics fall into type 1A according to Stebbins classification criteria. Cultivated species "Zunyi 3"possesses the same chromosome number and karyotype formula as "Bijie 7", while the ratio of chromosome length $(\mathrm{L} / \mathrm{S})$ is 1.69 , the constitution of the relative length is $4 \mathrm{~L}+8 \mathrm{M} 2+20 \mathrm{M} 1$. The relative lengths range from $9.66 \%-16.33 \%$, the centromeric index range from $37.22 \%-43.78 \%$, and arm ratio rankd from 1.28 to 1.69. Karyotype characteristics fall into type $1 \mathrm{~A}$.

\section{Introduction}

Cerasus pseudocerasus Lindl belongs to Prunoideae of Rosaceae, which originated from China and is widely distributed in Southwest and North China, including wild populations, landraces and large-scale cultivation varieties, their cultivation has a long history, is Chinese important and ancient germplasm [1-3].

Karyotype analysis is an important cytological research method that analyzes, compares, sorts and numbers chromosomes of an organism according to the length of chromosome, centromere position, arm ratio, Species evolution, classification and chromosomal structure, the relationship between the form and function of the essential means of an important and widely used in the evolution of species development and genetic relationship between species [4-5]. In this study, karyotype analysis was performed on two Chinese cherry landraces.

\section{Result and analysis}

"Bijie 7" chromosome karyotype.

The results of karyotype analysis of "Bijie 7" are shown in Table 1. The relative length of chromosomes ranged from $10.17 \%$ to $17.76 \%$. The relative length coefficient of chromosomes ranged from 0.81 to 1.42 , and the relative length of chromosomes was $4 \mathrm{~L}+8 \mathrm{M} 2+20 \mathrm{M} 1$. Fibroin index varied from $39.58 \%$ to $47.32 \%$, arm ratio ranged from 1.11 to 1.53 , and all 8 groups of chromosomes were centromeric chromosomes $(\mathrm{m})$. According to the classification criteria of Stebbins, the karyotype of Chinese cherry "Bijie 7" belongs to 1A type and nuclear type. The karyotype formula is $2 \mathrm{n}=4 \mathrm{x}=32=32 \mathrm{~m}$, chromosome length ratio is 1.75 and no arm ratio is greater than 2: 1. Type asymmetry coefficient of 57.26\%. The karyotype pattern shown in Figure 2 . 
Table 1 Chinese cherry "Bijie 7" chromosome karyotype parameters

\begin{tabular}{|c|c|c|c|c|c|c|c|}
\hline \multirow{2}{*}{$\begin{array}{l}\text { Number of } \\
\text { chromosome }\end{array}$} & \multicolumn{3}{|c|}{ Relative length/\% } & \multirow{2}{*}{$\begin{array}{l}\text { Relative length } \\
\text { factor }\end{array}$} & \multirow{2}{*}{$\begin{array}{l}\text { Centromere } \\
\text { index }\end{array}$} & \multirow{2}{*}{$\begin{array}{l}\text { Arm } \\
\text { ratio }\end{array}$} & \multirow[b]{2}{*}{ Type } \\
\hline & $\begin{array}{l}\text { Short } \\
\text { arm }\end{array}$ & $\begin{array}{c}\text { Long } \\
\text { arm }\end{array}$ & Full length & & & & \\
\hline 1 & 7.06 & 10.71 & 17.76 & 1.42 & 39.73 & 1.52 & $\mathrm{~m}$ \\
\hline 2 & 5.92 & 7.81 & 13.73 & 1.10 & 43.13 & 1.32 & $\mathrm{~m}$ \\
\hline 3 & 5.00 & 7.63 & 12.64 & 1.01 & 39.58 & 1.53 & $\mathrm{~m}$ \\
\hline 4 & 4.97 & 6.98 & 11.95 & 0.96 & 41.60 & 1.40 & $\mathrm{~m}$ \\
\hline 5 & 5.05 & 6.50 & 11.55 & 0.92 & 43.71 & 1.29 & $\mathrm{~m}$ \\
\hline 6 & 5.33 & 5.93 & 11.26 & 0.90 & 47.32 & 1.11 & $\mathrm{~m}$ \\
\hline 7 & 4.71 & 6.22 & 10.93 & 0.87 & 43.10 & 1.32 & $\mathrm{~m}$ \\
\hline 8 & 4.69 & 5.48 & 10.17 & 0.81 & 46.14 & 1.17 & $\mathrm{~m}$ \\
\hline
\end{tabular}

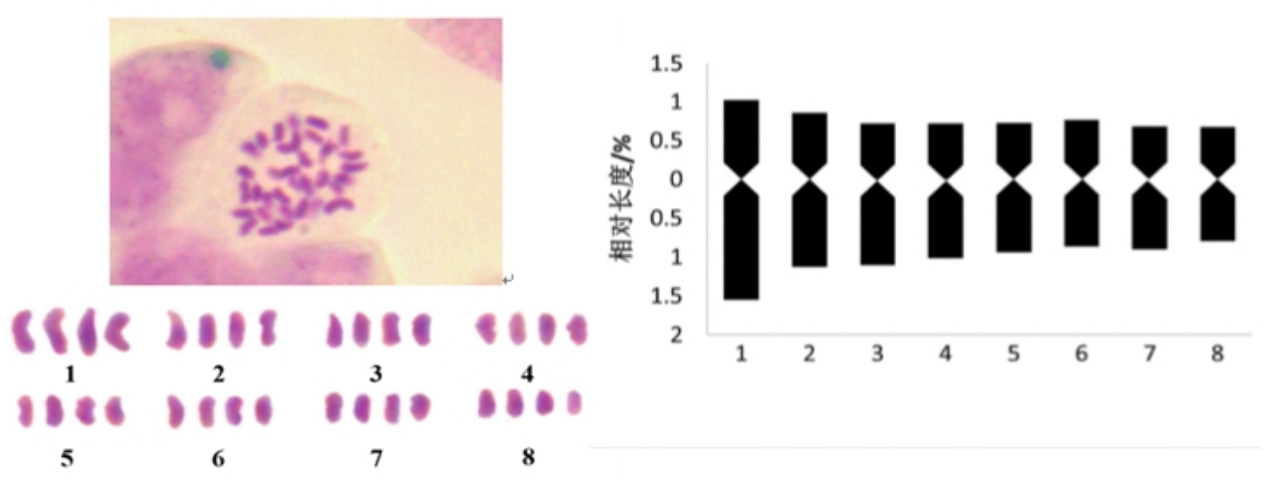

Fig.1 Karyotype of Chinese Cherry "Bijie 7" metaphase chromosomes

\section{"Zunyi 3" chromosome karyotype.}

The results of the "Zunyi 3" karyotype analysis are shown in Table 2. The relative lengths of the chromosomes ranged from $9.66 \%$ to $16.33 \%$. The relative length coefficient of chromosomes ranged from 0.77 to 1.31 and the relative length of chromosomes was 4L+8M2+20M1. Fibroin index varied from $37.22 \%$ to $43.78 \%$, arm ratio ranged from 1.28 to 1.69 , and all eight chromosomes were centromeric chromosomes $(\mathrm{m})$. According to the classification criteria of Stebbins, the karyotype of Chinese Zunyi 3 was $1 \mathrm{~A}$, and the karyotype was $2 \mathrm{n}=4 \mathrm{x}=32=32 \mathrm{~m}$. The chromosome length ratio was 1.69 and no arm ratio was greater than 2:1. Type asymmetry coefficient of $60.76 \%$. The karyotype pattern shown in Figure 2.

\section{Comparison of Karyotypes in Two Chinese Cherry Cultivars.}

According to the karyotype analysis of two Chinese cherry cultivars, the karyotype differences of "Bijie 7" and "ZunYi 3" were compared in this experiment, Comparison of nine karyotypes shown in Figure 3.

Chen et al. (1993) studied the karyotypes of Chinese cherries in Changli, Hebei Province. The results showed that the chromosome number of Chinese cherry was 8 , that of cultivar "Bijie 7 " and "Zunyi 3" was 32, which was tetraploid. Chromosomes are centromeric chromosomes (m). As early as 1931, Levitzky proposed that the evolution of flower plant karyotypes is enhanced with the enhancement of chromosome asymmetry. The karyotypes of two Chinese cherry cultivars were classified as $1 \mathrm{~A}$, indicating that the two had low degree of evolution. 
Table 2 Chinese cherry "Zunyi 3" chromosome karyotype parameters

\begin{tabular}{|c|c|c|c|c|c|c|c|}
\hline \multirow[b]{2}{*}{$\begin{array}{l}\text { Chromosome } \\
\text { length }\end{array}$} & \multicolumn{3}{|c|}{ Relatie length/\% } & \multirow{2}{*}{$\begin{array}{l}\text { Relative length } \\
\text { index }\end{array}$} & \multirow{2}{*}{$\begin{array}{l}\text { Centromere } \\
\text { index }\end{array}$} & \multirow[b]{2}{*}{$\begin{array}{l}\text { Arm } \\
\text { ratio }\end{array}$} & \multirow[b]{2}{*}{ Type } \\
\hline & $\begin{array}{c}\text { Short } \\
\text { arm }\end{array}$ & $\begin{array}{c}\text { Long } \\
\text { arm }\end{array}$ & Full length & & & & \\
\hline 1 & 6.88 & 9.45 & 16.33 & 1.31 & 42.15 & 1.37 & $\mathrm{~m}$ \\
\hline 2 & 5.41 & 8.88 & 14.30 & 1.14 & 37.86 & 1.64 & $\mathrm{~m}$ \\
\hline 3 & 4.81 & 8.11 & 12.92 & 1.03 & 37.23 & 1.69 & $\mathrm{~m}$ \\
\hline 4 & 4.60 & 7.76 & 12.36 & 0.99 & 37.22 & 1.69 & $\mathrm{~m}$ \\
\hline 5 & 4.57 & 7.39 & 11.96 & 0.96 & 38.21 & 1.62 & $\mathrm{~m}$ \\
\hline 6 & 4.41 & 7.11 & 11.52 & 0.92 & 38.26 & 1.61 & $\mathrm{~m}$ \\
\hline 7 & 4.32 & 6.63 & 10.95 & 0.88 & 39.46 & 1.53 & $\mathrm{~m}$ \\
\hline 8 & 4.23 & 5.43 & 9.66 & 0.77 & 43.78 & 1.28 & $\mathrm{~m}$ \\
\hline
\end{tabular}

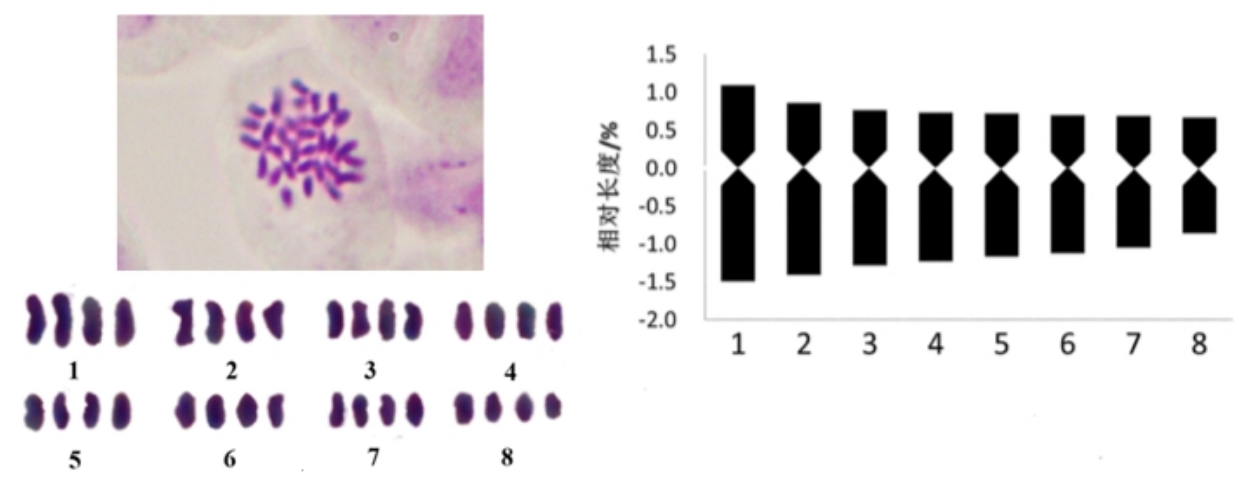

Fig.2 Karyotype of Chinese Cherry "Zunyi 3" metaphase chromosomes

Table 3 Comparison of karyotype differences between two Chinese cherry cultivars

\begin{tabular}{ccc}
\hline Karyotype index & Bijie7 & Zunyi 3 \\
\hline Chromosome number & $2 n=4 x=32$ & $2 n=4 x=32$ \\
Karyotype formula & $32 \mathrm{~m}$ & $32 \mathrm{~m}$ \\
Karyotype classification & $1 \mathrm{~A}$ & $1 \mathrm{~A}$ \\
Relative length range & $10.17-17.76$ & $9.66-16.33$ \\
Arm ratio range & $1.11-1.53$ & $1.28-1.69$ \\
Average arm ratio & 1.33 & 1.55 \\
Chromosome ratio with arm ratio $>2$ & 0 & 0 \\
Centromere index range & $39.58-47.32$ & $37.22-43.78$ \\
Karyotype asymmetry coefficient & 57.26 & 60.76 \\
\hline
\end{tabular}

\section{Materials and Methods}

Flower buds are from Sichuan Agricultural University teaching and research base (Chongzhou) for the material.

Fixed. Take the growing bud full of flowers, pall the scales of the outer package, so that the bud primordia can fully immersed in Carnoy's fixed solution (ethanol: glacial acetic acid $=3: 1$ ), fixed at $4{ }^{\circ} \mathrm{C} 24 \mathrm{~h}$, then $90 \%, 80 \% 70 \%$ ethanol gradient, and finally with $70 \%$ ethanol solution.

Chromosome spread. 70\% ethanol-treated flower buds were removed, washed with distilled water three times, each $10 \mathrm{~min}$. Then $60{ }^{\circ} \mathrm{C}$ water bath, with $1 \mathrm{~mol} / \mathrm{L} \mathrm{HCl}$ dissociation about $3 \mathrm{~min}$, immediately after soaking with distilled water washing 3 times, each $5 \mathrm{~min}$, remove the residual $\mathrm{HCl}$, 
in distilled water spare. Preparation of tablets using Acid solution method, dyeing by Carbol fuchsin. Chromosome selection and clear split phase observed with an Olympus BX-51 microscope, DP70 camera.

Data analysis. The karyotype analysis was performed using the criteria proposed by Li Maoxue and Chen Ruiyang [4]. The centromere position was determined. Based on the chromosome size and morphological characteristics, The relative length, arm ratio and type of chromosomes were calculated according to the nomenclature of Levan et al. [6]. The centromere index and the relative length coefficient of chromosomes were calculated according to the standard of Li Maoxue and Chen Ruiyang [4] Methods were calculated according to the criteria of Stebbins [7].

\section{Conclusions}

Chromosomes in Chinese cherries are smaller. Because the content of secondary metabolites in flower buds is higher than that of apical, the pretreatment of chromosomes in the experiment is more demanding. 8: 00-9: 00 in sunny day is better for sampling, if it is cloudy, the temperature is low, the sampling time should be slightly delayed.

In this study, chromosomes and karyotype analysis of two cultivated Chinese cherry cultivars collected from Guizhou Province were conducted. The results showed that the two cultivars of Chinese cherry were tetraploids. According to karyotype pairing, it was supposed to be an autotetraploid, which could be further analyzed by in situ hybridization to provide a cytological basis for breeding.

\section{Acknowledgements}

This research is supported by the National Natural Science Foundation of China (31272134).

\section{References}

[1] D.J. Yu, in: Chinese fruit tree taxonomy, edtied by Agricultural Press, Beijing, 1979, in press.

[2] D.J. Yu, C.N Li, in: Chinese Flora Vol.38, edtied by Science Press, Beijing, 1986, in press.

[3] C.J. Liu, M.Z Liu, in: Identification of Shanxi Houma bronze relics sites seeds, edtied by Heritage Press, Beijing, 1993, in press.

[4] M.X. Li, R.Y. Chen: About the standardization of plant karyotyping. Research of Botany in Wuhan, 1985, 3(4): 297-302.

[5] X.R. Wang, H.R. Tang, J. Duan, L Li: Journal of Plant Taxonomy. 2008, 46(4): 505-510.

[6] A. Levan, K. Fradga, A.A. Sandberg: Hereditas. 1964, 52: 197-201.

[7] G.L. Stebbins: Chromosomal evolution in higher plants. London: Edward Arnold Ltd. 1971: 87-89. 